\title{
Butterfly Effect: Tracing Shape Memory Effect and Elastic Bending in a Conformationally Flexible Organic Salt
}

\author{
Avantika Hasija ${ }^{1}$, S.R.N. Kiran Mangalampalli², Deepak Chopra*1 \\ ${ }^{1}$ Crystallography and Crystal Chemistry Laboratory, Department of Chemistry, Indian Institute of Science Education and Research, \\ Bhopal By-Pass Road, Bhopal, Madhya Pradesh 462 066, India. \\ ${ }^{2}$ Department of Physics and Nanotechnology, Faculty of Engineering and Technology, SRM Institute of Science and Technology, SRM \\ Nagar, Kattankulathur, Chennai, Kanchipuram, India. \\ avantika16@iiserb.ac.in
}

There are adequate number of molecules in nature which exhibit stimuli-response behaviour. Mimicking them, molecular crystals responding to mechanical and thermal stimuli, arraying actuating properties, comparable to that of soft materials, are yet in quest. With advances in the field of stimuli responsive molecular crystals, detailed investigation on the existing systems are helping in reformation of models which help in relating the macroscopic (kinematic) events to molecular (mechanistic) aspects.[1] Dynamic effects such as jumping, bending, popping, splitting as an outcome of thermal/mechanical/photo stimuli have become much more intriguing and explicate by linking observations from AFM/SEM/HSM to SCXRD/PXRD/Stress-Tensile test experimental data.[2,3]

On lowering temperature (258-278K), single crystals of a diphenyl phosphate 2-chloroanilium salt were observed exhibiting reversible thermal expansion/compression, [4] accompanying phase transition which simultaneously shows splitting and/or bending (morphology and size specific phenomenon) of crystals [5] (Fig 1, Right). The reversible thermoelastic phase transitioning behaviour could be classified under shape memory materials. [6,7] The elastic bending response to mechanical stimuli, on exerting force on the major face of the crystal at room temperature (Fig. 1, Left), highlights another exploitable application of this molecular crystal. [8]
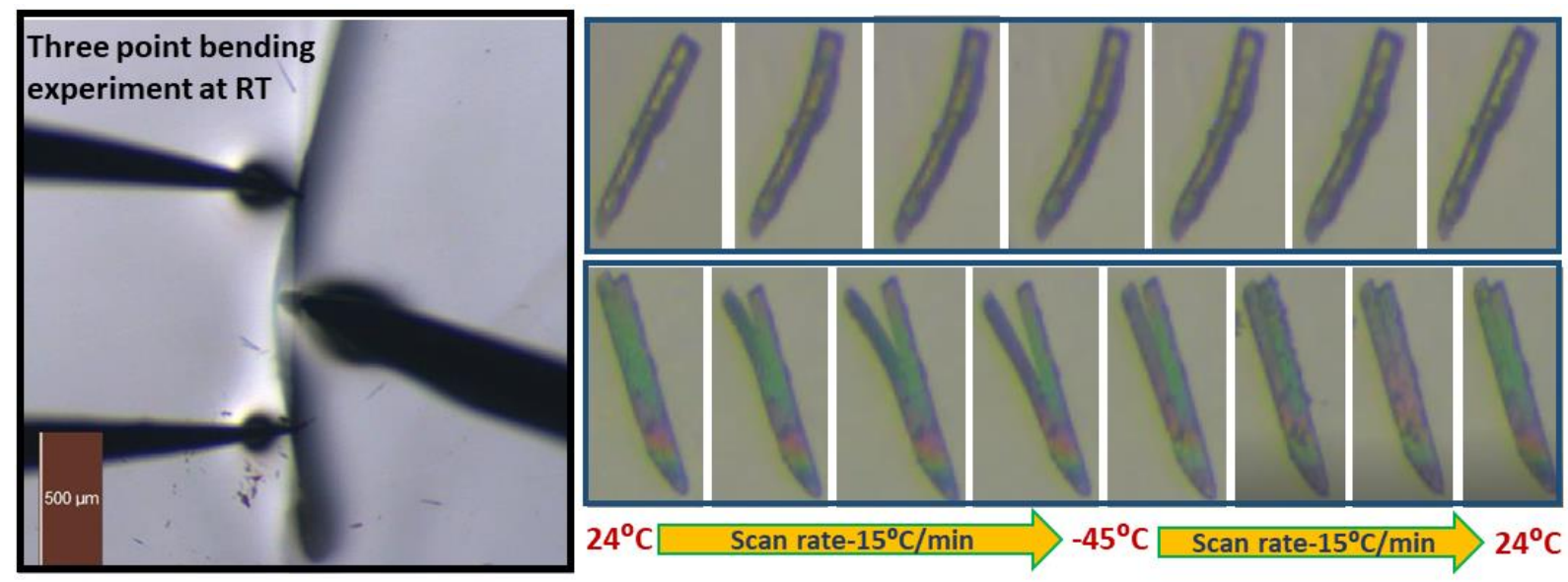

Figure 1. Three-point bending experiment carried out at major face (001) of single crystal; Observation of reversible bending and splitting on carrying out cooling-heating cycle of single crystals on cold stage microscope.

[1.] Karothu D. G,Weston J., Desta I.T. \& Naumov P. (2016). J. Am. Chem. Soc., 138, 13298-13306.

[2.] Devarapalli R., Kadambi S. B., Chen C-T, Rama Krishna G., Kammari B. R., Buehler M. J., Ramamurty U., \& Reddy C. M. (2019). Chem. Mater., 31, 1391-1402.

[3.] Dey S., Das S., Bhunia S., Chowdhury R., Mondal A., Bhattacharya B., Devarapalli R., Yasuda N., Moriwaki T., Mandal K., Mukherjee G. D. \& Reddy C. M. (2019). Nat. Commun., 10, 3711.

[4.] Liu H, Gutmann M. J., Stokes H. T., Campbell B. J., Evans I. R., \& Evans J.S.O. (2019). Chem. Mater., 31, 4514-4523.

[5.] Ahmed E., Karothu D. P., Warren M. \& Naumov P. (2019). Nat. Commun., 10, 3723.

[6.] Takamizawa S. \& Takasaki Y. (2016). Chem. Sci., 7, 1527-1534.

[7.] Park S. K. \& Diao Y. (2020). Chem. Soc. Rev., 49, 8287-8314.

[8.] Dey S., Das S., Bhunia S., Chowdhury R., Mondal A., Bhattacharya B., Devarapalli R., Yasuda N., Moriwaki T., Mandal K., Mukherjee G. D. \& Reddy C. M. (2019) Nat. Comm. 10, 3711.

Keywords: mechanically-thermally responsive; elastic bending; reversible phase transition; shape memory materials.

The authors thank IISER, Bhopal for Infrastructure and research facility. AH thank IISER, Bhopal for the doctoral fellowship. 